\title{
系列平行图的除 $V^{*}$ 外的边覆盖划分*
}

$$
\text { 刘桂真 }{ }^{* *}
$$

(山东大学数学与系统科学学院, 济南 250100)

\author{
邓小铁
}

(香港城市大学计算机系, 香港)

\author{
徐常青 \\ (河北工业大学应用数学系, 天津 300130)
}

\begin{abstract}
摘要 任意给定系列平行图 $G$ 的一个顶点 $v^{*}$, 则 $G$ 的边集可划分为 $k=\min$ $\left\{\kappa^{\prime}(G)+1, \delta(G)\right\}$ 个子集, 使得每一个边子集覆盖可能除 $v^{*}$ 以外的所有顶点, 其 中 $\delta(G)$ 为 $G$ 的最小度, $\kappa^{\prime}(G)$ 为 $G$ 的边连通度. 另外, 证明了该结果是最好的 可能，并且通过此证明过程得到一个可找到该划分的多项式时间算法.
\end{abstract}

\section{关键词 系列平行图 边连通度 边覆盖染色 极大 - 极小定理}

\section{1 引言}

边染色问题是将图的边集合划分为若干边子集, 使得每一个边子集中任意 两边没有公共顶点. 此类染色的目的是确定该种划分的最少子集数. 这一问题 在现实生活中有很多应用, 如网络设计和最优化问题等 ${ }^{[1]}$. 在计算机网络文件传 输问题中, 每台计算机 $x$ 具有 $f(x)$ 个通讯节点. 每对计算机间要进行一定数量 的文件传输. 在这种情形下考虑如何安排文件传输, 使得总传输过程所用时间最 短. 文件传输问题中如果每个文件的长度相同, 则可视为边染色问题 ${ }^{[1]}$. 该问题 可进行下述意义的推广, 要求划分的边子集具有其他的性质. 本文考虑如下形 式的边划分, 使得每一边子集覆盖可能除 $v^{*}$ 外的所有顶点, 其中 $v^{*}$ 是任一给定 顶点, 则该划分的目标是希望找到具有该性质的边子集的最大数目. 我们证明了 系列平行图的边集可划分为 $k=\min \left\{\kappa^{\prime}(G)+1, \delta(G)\right\}$ 个具有该性质的边子集.

* 国家自然科学基金 (批准号: 10471078)、高校博士点基金 (批准号: 20040422004) 和香港研究基金 
图与有向图关于极大 - 极小关系的研究已经取得了丰硕的成果, 该方面的研 究促进了图论和组合最优化的发展 ${ }^{[2]}$. 例如, Lucchesi 和 Younger 证明的著名的 极大 - 极小定理 ${ }^{[3,4]}$ 就是其中的结论之一: 任意给定一有向图 $D$, 它的不相交有 向割集的最大数目等于最小有向连接的基数, 其中 $D$ 的有向连接是与每一个有 向割集相交的弧所构成的子集. 相应的，1978 年 Woodall 猜想: 任一有向图的最 小有向割集的基数等于不交向连接的最大数目 (见文献 [5]). 文献 [6] 和 [7] 证明 了上面的猜想对有源和汇的连通有向图成立. 对平面图，通过对偶性，Woodall 猜想有下面的等价形式: 对任一有向平面图最短圈的边数等于不交横截的最大 基数目.

Lee 和 Wakabayashi 证明了 Woodall 的猜想对系列平行有向图成立 ${ }^{[8]}$. 文献 [9 11] 证明了对任一简单图 $G$, 不交边覆盖的最大数目等于 $\delta(G)$ 或 $\delta(G)-1$. 注 意到该结果对多重图不成立. Seymour 在文献 [12] 中证明了一类 “极大 - 极小公 式”对系列平行图的色数成立. Zhou 等人给出系列平行图边染色的一个线性算 法 (见文献 [13]). 该方面的有关结果见文献 [14, 15, 5].

本文考虑与文献 [8] 和 [11] 中结果相关的系列平行多重图的问题. 文中给 出相关的定义和记号以及主要定理, 即在系列平行图中可确定 $k=\min \left\{\kappa^{\prime}(G)+\right.$ $1, \delta(G)\}$ 个边覆盖子集 (可能不包括顶点 $v^{*}$ ) 并说明这一结论是最好的. 我们对结 果进行了讨论, 给出对结果的说明和一些新的猜想.

显然通过证明过程可获得一个找到该划分的多项式时间算法. 在第 4 节中 将证明对于任意的图确定边覆盖划分 (可能不包括顶点 $v^{*}$ ) 的最大基数是 $N P$ 困 难的. 尽管我们的证明依赖系列平行图的结构并不能直接推广到平面图, 但我 们猜想平面图也具有该性质, 并且可以证明该性质蕴含着四色定理. 此外, 我们 还给出关于平面图边覆盖划分的另一猜想.

\section{2 定义及结果}

用 $G=(V, E)$ 表示具有顶点集 $V(G)$ 和边集 $E(G)$ 的图. 本文所考虑的图 为重图, 即任一对顶点间可能有两条或更多条边相连. 对于顶点 $v \in V(G)$, 顶 点 $v$ 的度 (与其关联的边数) 记为 $d_{G}(v)$. 令 $\delta(G)$ 和 $\kappa^{\prime}(G)$ 分别表示图 $G$ 的最小 度和边连通度. 对于 $V(G)$ 的任一子集 $S$, 由 $S$ 导出的图 $G$ 的子图记为 $G[S]$. 子 图 $G[V(G) \backslash S]$ 记为 $G-S$. 对 $V(G)$ 的任意两个不交子集 $S$ 和 $T$, 令 $E_{G}(S, T)=$ $\{x y \mid x y \in E(G), x \in S$ 且 $y \in T\}$.

图 $G$ 中的链 $L=\left(v_{1}, e_{1}, v_{2}, \cdots, v_{m-1}, e_{m-1}, v_{m}\right)$ 是一顶点与边的交错序列, 满足当 $1 \leqslant i \leqslant m-1$ 时边 $e_{i}$ 的端点为 $v_{i}$ 和 $v_{i+1}$ 并且边 $e_{1}, e_{2}, \cdots, e_{m-1}$ 互异. 链 $L=\left(v_{1}, e_{1}, v_{2}, \cdots, v_{m-1}, e_{m-1}, v_{m}\right)$ 的子链是 $L$ 中的一段连续部分 $\left(v_{i}, e_{i}, v_{i+1}, \cdots\right.$, $\left.v_{j-1}, e_{j-1}, v_{j}\right)$. 如果链 $L$ 中的顶点 $v_{1}, v_{2}, \cdots, v_{m}$ 互异, 则 $L$ 称为路. 一个圈是满 足 $v_{1}=v_{m}$, 并且当 $\{i, j\} \neq\{1, m\}$ 时, $v_{i} \neq v_{j}$ 的链. 为简单起见, 仅用链的顶 
点 $L=\left(v_{1}, v_{2}, \cdots, v_{m-1}, v_{m}\right)$ 表示链 $L$.

系列平行图是不含 $K_{4}$ (4 个顶点的完全图) 的剖分作为子图的图. 令 $\mathcal{P}=$ $\left(E_{1}, E_{2}, \cdots, E_{k}\right)$ 为 $E(G)$ 的一个划分, $d_{i}(v)$ 表示 $v$ 在由 $E_{i}$ 导出的 $G$ 的子图 $G\left[E_{i}\right]$ 中的度, $i=1, \cdots, k$. 如果对于任一 $v \in V(G), v \neq v^{*}$ 都有 $d_{i}(v) \geqslant 1$, 则称 $E_{i}$ 是 $v^{*}$ - 排除边覆盖. 如果每一个 $E_{i}$ 都是 $v^{*}$ - 排除边覆盖 $(i=1, \cdots, k)$, 则称划 分 $\mathcal{P}=\left(E_{1}, E_{2}, \cdots, E_{k}\right)$ 是图 $G$ 的 $v^{*}$ - 排除边覆盖划分.

本文将系列平行图的边集划分为尽可能多的 $v^{*}$ - 排除边覆盖, 即对任一系列 平行图 $G$ 和任一给定的顶点 $v^{*} \in V(G)$, 确定图 $G$ 的具有最大基数的 $v^{*}$ - 排除边覆 盖划分 $\mathcal{P}$ 的基数 $|\mathcal{P}|$ 的下界. 我们证明了对系列平行图 $k=\min \left\{\kappa^{\prime}(G)+1, \delta(G)\right\}$ 是 $|\mathcal{P}|$ 的最好下界.

从另一观点来研究该问题是考察其对偶问题. 一个平面图的面如果其边界 所含边数最小, 则称其为平面图的最小面. 令 $E_{i}$ 表示 $E(G)$ 的子集, $f^{*}$ 为一给 定面. 如果 $E_{i}$ 至少含有 $f$ 边界上的一条边, 则称 $E_{i}$ 与面 $f$ 相交. 边子集 $E_{i}$ 称 为 $f^{*}$ - 排除面横截, 如果 $E_{i}$ 与 $f^{*}$ 外的所有面相交. 如果 $\mathcal{P}=\left(E_{1}, E_{2}, \cdots, E_{k}\right)$ 是 $E(G)$ 的一个划分并且每一个 $E_{i}$ 都是 $f^{*}$ - 排除面横截 $(1 \leqslant i \leqslant k)$, 则称 $\mathcal{P}$ 是一个 $f^{*}$ - 排除面横截的装箱, $|\mathcal{P}|$ 的下界为 $\min \{g(G)+1, c(G)\}$, 其中 $g(G)$ 是图 $G$ 的 围长, $c(G)$ 是最小面边界所含边数, $\mathcal{P}$ 是具有最大基数的图 $G$ 的 $f^{*}$ - 排除面横 截之装箱.

我们的主要结论是定理 2.1 和 2.2.

定理 2.1 设图 $G$ 为系列平行图, $\kappa^{\prime}(G)$ 为其边连通度, 则对任一给定的图 $G$ 的顶点 $v^{*}$, 边集 $E(G)$ 可划分为 $\min \left\{\kappa^{\prime}(G)+1, \delta(G)\right\}$ 个 $v^{*}$ - 排除边覆盖.

定理 2.2 设图 $G$ 为系列平行图的平面嵌入图, 则对任一给定的图 $G$ 的面 $f^{*}$, 存在基数为 $\min \{g(G)+1, c(G)\}$ 的 $f^{*}$ - 排除面横截装箱.

注意到系列平行图的对偶仍然是系列平行图，在对偶图中割集变为圈，所 以上述两个定理是等价的.

注释 2.1 易知上述两个定理中的界都是最好的并且定理中的条件 “ $v^{*}$ - 排 除” 和 “ $f^{*}$ - 排除” 是不可去的. 例如: 设图 $G$ 为一系列平行图, 满足 $\kappa^{\prime}(G)+1 \geqslant \delta(G)$ 并且至少有两个最小度顶点, 则对于任一给定的顶点 $v^{*}, E(G)$ 至多可以划分为 $\delta(G)$ 个 $v^{*}$ - 排除边覆盖. 另外, 易验证存在许多图 $G$ 满足 $\kappa^{\prime}(G)+1<\delta(G)$ (例 如, 一个 3 -正则系列平行图 $G$, 有 $\kappa^{\prime}(G)=1$ 和 3 条割边), 则 $E(G)$ 恰好可以划分 为 $\kappa^{\prime}(G)+1<\delta(G)$ 个 $v^{*}$ - 排除边覆盖, 所以下界 $\kappa^{\prime}(G)+1$ 是最好的并且不能被 $\delta(G)$ 替代. 对于任一奇数阶的 $r$ 边连通 $r$-正则系列平行图 $G, E(G)$ 可以划分成 $k=\delta(G)$ 个 $v^{*}$ - 排除边覆盖, 但不能划分成 $k=\delta(G)$ 个边覆盖. 由上述讨论知 我们的结果是最好的.

注释 2.2 当 $\kappa^{\prime}(G)+1 \geqslant \delta(G)$ 或 $g(G)+1 \geqslant c(G)$, 并且 $G$ 为 $\delta(G)$ - 边连通 图或 $G$ 的一个最小圈作为平面图的一个内部面时, 上面两个定理中不等式的等 
号成立. 从这个意义上讲我们证明了下面的极大极小关系:

推论 2.1 设图 $G$ 为一系列平行图. 如果边连通度 $\kappa^{\prime}(G) \geqslant \delta(G)-1$, 则对 $G$ 中任一选定的顶点 $v^{*}$, 图 $G$ 的不交 $v^{*}$ - 排除边覆盖的最大数目等于图 $G$ 的最小 度.

推论 2.2 设图 $G$ 为系列平行图的平面嵌入图, 围长 $g(G) \geqslant c(G)-1$, 则对 任一给定的 $G$ 中的面 $f^{*}$, 图 $G$ 的不交 $f^{*}$ - 排除面横截的最大基数等于图 $G$ 的最 小面所含边数.

另一方面, 一些图的不交 $v^{*}$ - 排除边覆盖数可能远大于 $\kappa^{\prime}(G)$. 第 4 节将给出 一个关于其确切值的猜想.

\section{3 主要定理的证明}

由文献 [16] 可知, 边集 $E(G)$ 的一个划分 $\left(E_{1}, E_{2}, \cdots, E_{k}\right)$ 可以看成是一个 $k$ - 边染色, 使得 $E_{i}$ 中的边染颜色 $i$. 一个 $k$ - 边覆盖染色就是 $\left(E_{1}, E_{2}, \cdots, E_{k}\right)$ 的 $k$ - 边染色中每一个 $E_{i}$ 都是一个边覆盖. 类似的, 一个几乎 $k$-边覆盖染色是 指 $\left(E_{1}, E_{2}, \cdots, E_{k}\right)$ 的 $k$ - 边染色中每一个 $E_{i}$ 都覆盖图 $G$ 的可能除 $v^{*}$ 外的所有顶 点. 与 $v$ 关联的某边染颜色 $i$, 就称颜色 $i$ 在顶点 $v$ 出现. 链 $L=\left(v_{1}, e_{1}, v_{2}, \cdots, v_{m-1}\right.$, $\left.e_{m-1}, v_{m}\right)$ 称为 $(a, b)$ - 交错链, 如果 $L$ 中的边交替染颜色 $a$ 与 $b$ 并且第 1 条边 $e_{1}$ 染 $a$ 色, 称 $e_{1}$ 为 $L$ 的起始边, $e_{m}$ 为终止边. 交换 $L$ 中边所染颜色 $a$ 与 $b$ 称为切 换 $L$. 给定图 $G$ 的一个 $k$-边染色 $\mathcal{C}$, 用 $c(v)$ 表示在 $v$ 点出现的互异颜色数, 显然 有 $c(v) \leqslant d_{G}(v)$. 称 $k$-边染色 $\mathcal{C}^{\prime}$ 是 $\mathcal{C}$ 的改进, 如果 $\sum_{v \in V(G)} c^{\prime}(v)>\sum_{v \in V(G)} c(v)$, 其中 $c^{\prime}(v)$ 是染色 $\mathcal{C}^{\prime}$ 中顶点 $v$ 出现的互异颜色数. 如果一 $k$ - 边染色不能再改进, 则称其为最优 $k$-边染色. 令 $\mathcal{C}=\left(E_{1}, E_{2}, \cdots, E_{k}\right)$ 为图 $G$ 的最优 $k$-边染色. 对任 意的 $i, 1 \leqslant i \leqslant k$, 且 $v \neq v^{*}$, 定义

$$
f(\mathcal{C}, v)=\mid\left\{E_{i} \mid E_{i} \in \mathcal{C} \text { 且 } d_{i}(v)=0\right\} \mid
$$

且

$$
f(\mathcal{C})=\sum_{v \neq v^{*}} f(\mathcal{C}, v),
$$

则 $\mathcal{C}$ 是几乎 $k$-边覆盖染色当且仅当 $f(\mathcal{C})=0$. 我们用边染色证明定理 2.1. 在证 明过程中下面两个引理起着重要的作用:

引理 3.1(文献 [16] 中的引理 6.1.1) 设图 $G$ 为不是奇圈的连通图, 则 $G$ 存 在一个 2 - 边染色, 使得每种颜色在每一个顶点处均出现.

引理 3.2(文献 [16] 中的引理 6.1.2) 设 $\mathcal{C}=\left(E_{1}, E_{2}, \cdots, E_{k}\right)$ 为图 $G$ 的一个 最优 $k$-边着色. 若存在图 $G$ 中的顶点 $u$ 及颜色 $i$ 和 $j$, 使得 $i$ 在 $u$ 不出现而 $j$ 至 少在 $u$ 出现两次, 则含 $u$ 点的分支 $G\left[E_{i} \cup E_{j}\right]$ 为一奇圈.

定理 2.1 的证明 令 $k=\min \left\{\kappa^{\prime}(G)+1, \delta(G)\right\}$. 本证明的主要思想是确定一 个具有特殊性质的最优 $k$ - 边染色 $\mathcal{C}$. 进一步证明 $\mathcal{C}$ 是几乎 $k$ - 边覆盖染色, 亦即 
每种颜色在 $V(G) \backslash\left\{v^{*}\right\}$ 中的每一顶点处均出现, 然后推出矛盾.

当 $k=1$ 时, 显然定理成立. 当 $k=2$ 时, 由引理 3.1 知定理成立. 现在考虑 $k \geqslant 3$ 的情形. 设 $\mathcal{C}=\left(E_{1}, E_{2}, \cdots, E_{k}\right)$ 是图 $G$ 的一个最优 $k$ - 边染色，使得 $f(\mathcal{C})$ 最小, 即对所有的最优 $k$ - 边染色 $\mathcal{C}^{\prime}$, 有 $f(\mathcal{C}) \leqslant f\left(\mathcal{C}^{\prime}\right)$, 则称 $\mathcal{C}$ 为最小 $k$-边染色. 如果 $f(\mathcal{C}) \geqslant 1$, 则必存在顶点 $v_{1} \neq v^{*}$ 和颜色 $i_{0}$, 使得 $i_{0}$ 在 $v_{1}$ 不出现, 亦即在 $\mathcal{C}$ 中 $d_{i_{0}}\left(v_{1}\right)=0$. 因为 $d_{G}\left(v_{1}\right) \geqslant \delta(G) \geqslant k$, 必存在颜色 $i_{1}$, 使得 $i_{1}$ 至少在 $v_{1}$ 出现两 次, 即 $d_{i_{1}}\left(v_{1}\right) \geqslant 2$. 由引理 3.2 知, 含 $v_{1}$ 的连通分支 $G\left[E_{i_{0}} \cup E_{i_{1}}\right]$ 为一奇圈 $C_{1}$. 令 $\alpha\left(C_{1}, v^{*}, \mathcal{C}\right)$ 表示 $G-V\left(C_{1}\right)$ 中包含 $v^{*}$ 的连通分支所含顶点数. 如果 $v^{*} \in V\left(C_{1}\right)$, 规定 $\alpha\left(C_{1}, v^{*}, \mathcal{C}\right)=0$. 选取一个最小 $k$-边染色 $\mathcal{C}$ 和 $C_{1}$, 使得 $\alpha\left(C_{1}, v^{*}, \mathcal{C}\right)$ 最小, 即 对任一最小 $k$-边染色 $\mathcal{C}^{\prime}$ 和任一上述交错奇圈 $C_{1}^{\prime}, \alpha\left(C_{1}, v^{*}, \mathcal{C}\right) \leqslant \alpha\left(C_{1}^{\prime}, v^{*}, \mathcal{C}^{\prime}\right)$. 可 以证明 $\mathcal{C}$ 即为所求. 假设

$$
C_{1}=\left(v_{1}, e_{1}, v_{2}, \cdots, v_{2 l-1}, e_{2 l-1}, v_{2 l}=v_{1}\right),
$$

其中 $e_{2 i-1}$ 染颜色 $i_{1}, e_{2 i}$ 染颜色 $i_{0}, 1 \leqslant i \leqslant l$. 我们考虑下面 3 种情形:

情形 1 存在 $C_{1}$ 中一个顶点 $v_{i}$, 使得 $v_{i}=v^{*}$.

在这种情形下通过切换路 $L_{1}=\left(v_{1}, e_{1}, v_{2}, \cdots, v_{i-1}, e_{i-1}, v_{i}\right)$, 得到图 $G$ 一个 新的 $k$ - 边染色 $\mathcal{C}^{\prime}=\left(E_{1}^{\prime}, E_{2}^{\prime}, \cdots, E_{k}^{\prime}\right)$. 易证

$$
c^{\prime}\left(v_{1}\right)=c\left(v_{1}\right)+1, \quad c^{\prime}\left(v^{*}\right)=c\left(v^{*}\right)-1,
$$

并且对所有的 $v \neq v_{1}, v^{*}$, 有 $c^{\prime}(v)=c(v)$, 故 $\mathcal{C}^{\prime}$ 为图 $G$ 的最小 $k$-边染色, 且 $f\left(\mathcal{C}^{\prime}\right)<f(\mathcal{C})$, 矛盾.

现在假设 $v^{*} \notin C_{1}$. 令 $H$ 表示 $G-V\left(C_{1}\right)$ 中含 $v^{*}$ 的连通分支, 则 $C_{1}$ 中至多 有两个点与 $H$ 关联, 否则, 图 $G$ 中将出现 $K_{4}$ 的剖分.

情形 $2 C_{1}$ 中仅有一个顶点 $v_{i}$ 与 $H$ 关联.

令 $S=E_{G}\left(V\left(C_{1}\right), V(H)\right)$. 因为 $\kappa^{\prime}(G)+1 \geqslant k$, 有 $|S| \geqslant \kappa^{\prime}(G) \geqslant k-1$. 注意到 $\mathcal{C}$ 中 $d_{i_{0}}\left(v_{i}\right)+d_{i_{1}}\left(v_{i}\right)=2$ 并且 $k \geqslant 3$, 则必存在一种颜色 $i_{2}$, 使得 $S$ 中的边 $e=v_{i} v_{p}$ 和 $e^{\prime}=v_{i} v_{q}$ 均染颜色 $i_{2}$. 通过切换路 $L=\left(v_{1}, e_{1}, v_{2}, \cdots, v_{i-1}, e_{i-1}, v_{i}\right)$, 我们得到 图 $G$ 的一个新 $k$-边染色 $\mathcal{C}^{\prime}=\left(E_{1}^{\prime}, E_{2}^{\prime}, \cdots, E_{k}^{\prime}\right)$ (如果 $v_{i}=v_{1}$, 则 $\mathcal{C}^{\prime}=\mathcal{C}$ ). 不失一般 性, 假设边 $e_{i-1}$ 在 $\mathcal{C}$ 中染颜色 $i_{0}$, 故在 $\mathcal{C}^{\prime}$ 中颜色 $i_{0}$ 在 $v_{i}$ 不出现而颜色 $i_{2}$ 在 $v_{i}$ 至 少出现两次. 容易验证

$$
c^{\prime}\left(v_{1}\right)=c\left(v_{1}\right)+1, \quad c^{\prime}\left(v_{i}\right)=c\left(v_{i}\right)-1,
$$

并且对所有的 $v \neq v_{1}, v_{i}$, 有 $c^{\prime}(v)=c(v)$, 从而 $\mathcal{C}^{\prime}$ 也是图 $G$ 的最小 $k$-边染色. 由引 理 3.2 知 $G\left[E_{i_{0}} \cup E_{i_{2}}\right]$ 的含 $v_{i}$ 的连通分支 $C_{2}$ 为一奇圈. 如果 $v^{*} \in V\left(C_{2}\right)$, 则归结 为情形 1 , 推出矛盾. 否则, $G-V\left(C_{2}\right)$ 的含 $v^{*}$ 的连通分支 $H_{1}$ 为 $H$ 的真子集, 即 $V\left(H_{1}\right) \subset V(H)$, 与 $\alpha\left(C_{1}, v^{*}, \mathcal{C}\right)$ 最小矛盾.

情形 $3 C_{1}$ 中有两个顶点 $v_{i}$ 和 $v_{j}$ 与 $H$ 关联.

不妨假设 $i<j$ 并且在 $\mathcal{C}$ 中边 $e_{i-1}=v_{i-1} v_{i}$ 染颜色 $i_{0}$. 令 $S=E\left(V\left(C_{1}\right), V(H)\right)$, 用 $E(v)$ 表示 $S$ 中与 $v$ 关联的边集. 通过切换 $L_{1}=\left(v_{1}, e_{1}, v_{2}, \cdots, v_{i-1}, e_{i-1}, v_{i}\right)$, 
得到图 $G$ 的一个新 $k$-边染色 $\mathcal{C}^{\prime}=\left(E_{1}^{\prime}, E_{2}^{\prime}, \cdots, E_{k}^{\prime}\right)$, 满足

$$
c^{\prime}\left(v_{1}\right)=c\left(v_{1}\right)+1, \quad c^{\prime}\left(v_{i}\right)=c\left(v_{i}\right)-1,
$$

并且对所有的 $v \neq v_{1}, v_{i}$, 有 $c^{\prime}(v)=c(v)$, 故 $\mathcal{C}^{\prime}$ 也是图 $G$ 的最小 $k$-边染色 (如果 $v_{i}=v_{1}$, 则 $\mathcal{C}^{\prime}=\mathcal{C}$ ). 类似于情形 2 , 易知在 $\mathcal{C}^{\prime}$ 中至少有 $S$ 的两条边染相同颜色. 设 $\mathcal{C}^{\prime}$ 中 $S$ 的边 $e=v_{i} v_{p} \in E\left(v_{i}\right)$ 和 $f$ 染相同颜色 $i_{2} \neq i_{0}, i_{1}$. 注意到边 $e_{i}$ 和 $e_{i-1}$ 在 $\mathcal{C}^{\prime}$ 中染颜色 $i_{1}$. 现在考虑 $G\left[E_{i_{1}}^{\prime} \cup E_{i_{2}}^{\prime}\right]$ 的含 $v_{i}$ 的分支 $G_{12}$. 在 $G_{12}$ 中选取以 $e_{i}=v_{i} v_{i+1}$ 作为起始边的最短 $\left(i_{1}, i_{2}\right)$ - 交错链 $L_{2}=\left(v_{i}, v_{i+1}, u_{1}, u_{2}, \cdots, u_{m-1}, u_{m}\right)$, 使得切换 链 $L_{2}$ 后仍然得到最小 $k$ - 边染色. 因为 $\mathcal{C}^{\prime}$ 是图 $G$ 的最小 $k$-边染色, 故 $v^{*} \notin L_{2}$. 简 单起见, 当一个链切换后没有增加 $f(\mathcal{C})$, 则称其为不增链. 易知总存在不增链. 分两种情形讨论.

情形 3.1 在 $G_{12}$ 中存在一个最短 $\left(i_{1}, i_{2}\right)$ - 交错路 $L_{2}=\left(v_{i}, v_{i+1}, u_{1}, u_{2}, \cdots\right.$, $\left.u_{m-1}, u_{m}\right)$, 满足 $f_{m-1}=u_{m-1} u_{m}$ 染颜色 $i_{1}\left(i_{2}\right)$ 并且在 $\mathcal{C}^{\prime}$ 中 $d_{i_{2}}\left(u_{m}\right)=0$ 或 $d_{i_{1}}\left(u_{m}\right) \geqslant 2$ (在 $\mathcal{C}^{\prime}$ 中 $d_{i_{1}}\left(u_{m}\right)=0$ 或 $d_{i_{2}}\left(u_{m}\right) \geqslant 2$ ).

在该情形下易知 $L_{2}$ 为一不增路. 切换路 $L_{2}$ 得到一个最小 $k$-边染色 $\mathcal{C}^{\prime \prime}=$ $\left(E_{1}^{\prime \prime}, E_{2}^{\prime \prime}, \cdots, E_{k}^{\prime \prime}\right)$, 使得边 $e_{i}=v_{i} v_{i+1}$ 染颜色 $i_{2}$. 因为 $L_{2}$ 是以 $v_{i}$ 为起点的路, 说 明边 $e=v_{i} v_{p}$ 不在 $L_{2}$ 上，从而在 $\mathcal{C}^{\prime \prime}$ 中边 $e$ 仍然染颜色 $i_{2}$. 故在 $\mathcal{C}^{\prime \prime}$ 中颜色 $i_{0}$ 在 $v_{i}$ 不出现, 颜色 $i_{2}$ 在 $v_{i}$ 至少出现两次. 由引理 3.2, $G\left[E_{i_{0}}^{\prime \prime} \cup E_{i_{2}}^{\prime \prime}\right]$ 的含 $v_{i}$ 的连通 分支 $C_{2}$ 为一奇圈. 显然, 圈 $C_{2}$ 含边 $e=v_{i} v_{p}$ 和 $e_{i}=v_{i} v_{i+1}$. 因为 $C_{1}$ 中只有两 个顶点与 $H$ 关联又 $v_{p} \in V(H)$, 则 $v_{j}$ 必在 $C_{2}$ 上. 如果 $v^{*} \in V\left(C_{2}\right)$, 则情形 1 出 现, 可得矛盾. 否则, $G-V\left(C_{2}\right)$ 的含 $v^{*}$ 的连通分支 $H_{1}$ 是 $H$ 的真子集, 易即 $V\left(H_{1}\right) \subset V(H)$, 与 $\alpha\left(C_{1}, v^{*}, \mathcal{C}\right)$ 的最小性矛盾.

情形 3.2 在 $G_{12}$ 中存在一条最短 $\left(i_{1}, i_{2}\right)$ - 交错链 $L_{2}=\left(v_{i}, v_{i+1}, u_{1}, u_{2}, \cdots\right.$, $\left.u_{n}, \cdots, u_{m-1}, u_{m}\right)$, 使得 $u_{n}=u_{m}$ 并且其他顶点互异.

情形 3.2.1 边 $e=v_{i} v_{p}$ 和 $e_{i-1}=v_{i-1} v_{i}$ 均不在 $L_{2}$ 中.

在该情形下 $L_{2}$ 含唯一的圈 $C=\left(u_{n}, \cdots, u_{m}\right)$ 并且易知 $L_{2}$ 为不增链. 顶点 $u_{n}$ 可能等于 $v_{i}$. 显然, 通过切换 $L_{2}$ 我们得到一个新的 $k$-边染色 $\mathcal{C}^{\prime \prime}=\left(E_{1}^{\prime \prime}, E_{2}^{\prime \prime}, \cdots, E_{k}^{\prime \prime}\right)$, 使得边 $e_{i}=v_{i} v_{i+1}$ 染颜色 $i_{2}$. 因为 $e=v_{i} v_{p} \notin L_{2}$, 在 $\mathcal{C}^{\prime \prime}$ 中颜色 $i_{0}$ 在 $v_{i}$ 不出现并 且颜色 $i_{2}$ 在 $v_{i}$ 至少出现两次, 从而顶点 $v_{i}$ 满足引理 3.2 中的条件. 同情形 3.1 得 到矛盾.

情形 3.2.2 边 $e=v_{i} v_{p} \in L_{2}, e_{i-1}=v_{i-1} v_{i} \notin L_{2}$.

此时因为 $L_{2}$ 是最短链, 知 $L_{2}=C$ 是一个圈且 $u_{n}=u_{m}=v_{i}$. 切换链 $L_{2}$ 得 一个最小 $k$ - 边染色 $\mathcal{C}^{\prime \prime}=\left(E_{1}^{\prime \prime}, E_{2}^{\prime \prime}, \cdots, E_{k}^{\prime \prime}\right)$, 其中 $e_{i}=v_{i} v_{i+1}$ 染颜色 $i_{2}$, 边 $e=v_{i} v_{p}$ 染颜色 $i_{1}$. 注意到在 $\mathcal{C}^{\prime \prime}$ 中 $e_{i-1}=v_{i} v_{i-1}$ 仍染颜色 $i_{1}$, 故在 $\mathcal{C}^{\prime \prime}$ 中颜色 $i_{0}$ 在 $v_{i}$ 不出 现, 颜色 $i_{1}$ 至少在 $v_{i}$ 出现两次, 从而顶点 $v_{i}$ 亦满足引理 3.2 的条件, $G\left[E_{i_{0}}^{\prime \prime} \cup E_{i_{1}}^{\prime \prime}\right]$ 中含顶点 $v_{i}$ 的连通分支必为奇圈. 类似于情形 3.2.1 可推出矛盾.

情形 3.3 边 $e_{i-1}=v_{i-1} v_{i}$ 在 $L_{2}$ 上. 
注意到此时 $L_{2}$ 含圈 $C_{2}=\left(v_{i}, v_{i+1}, \cdots, v_{i-1}, v_{i}\right)$ 且 $C_{2}$ 是不增链. 当切换 $C_{2}$ 后, 颜色 $i_{1}$ 不在顶点 $v_{i}$ 出现. 分两种情形讨论.

情形 3.3.1 在 $\mathcal{C}^{\prime}$ 中存在一条边 $f=v_{j} v_{q} \in E\left(v_{j}\right)$ 染颜色 $i_{2}$.

(a) $v_{i+1}=v_{j}$.

此时 $L_{2}$ 的子链: 交错路 $L_{2}^{\prime}=\left(v_{j}=v_{i+1}, \cdots, v_{i-1}, v_{i}\right)$ 为一不增链. 通过切 换 $L_{2}^{\prime}$, 得到一个最小 $k$-边染色 $\mathcal{C}^{\prime \prime}$, 其中 $e_{i}=v_{i} v_{j}$ 仍染颜色 $i_{1}$ 并且边 $e=v_{i} v_{p}$ 和 $e_{i-1}=v_{i-1} v_{i}$ 均染颜色 $i_{2}$, 故顶点 $v_{i}$ 满足引理 3.2 的条件. $G\left[E_{i_{0}}^{\prime \prime} \cup E_{i_{2}}^{\prime \prime}\right]$ 的含 $v_{i}$ 的连通分支为一奇圈, 该奇圈含顶点 $v_{j}$. 类似于情形 3.2.1 推出矛盾.

(b) $v_{i+1} \neq v_{j}$.

此时我们有下述断言:

断言 1 顶点 $v_{j} \in V\left(L_{2}\right)$.

若否, $v_{j} \notin V\left(L_{2}\right)$. 因为点 $v_{i+1}, v_{i}$ 和 $v_{i-1}$ 均在 $L_{2}$ 中, 所以存在 $L_{2}$ 的一个 子链 $P_{1}$, 使得 $P_{1}$ 是一条连接顶点 $v_{i+1}$ 和 $v_{s}$ 的路, 其中 $s>j$ 或 $s \leqslant i-1$. 因为 $H$ 连通, 在 $H$ 中存在连接 $v_{q}$ 和 $v_{p}$ 的路 $P^{\prime}$, 故 $P=f \cup P^{\prime} \cup e$ 是 $G$ 中连接 $v_{j}$ 和 $v_{i}$ 的 路. 易知 $C_{1}, P_{1}$ 和 $P$ 构成图 $G$ 的 $K_{4}$ 剖分, 与 $G$ 为系列平行图矛盾. 断言成立.

此时如果 $L_{2}$ 中的边 $v_{j^{\prime}} v_{j}$ 具有颜色 $i_{1}\left(j^{\prime}<j\right)$, 则同情形 $\mathrm{a}$ 的证明可推出矛 盾. 如果 $L_{2}$ 中的边 $v_{j^{\prime}} v_{j}$ 具有颜色 $i_{2}\left(j^{\prime}<j\right)$, 则 $L_{2}$ 的子链 $L_{2}^{\prime}=\left(v_{i}, v_{i+1}, \cdots, v_{j}\right)$ 为交错路, 并且是不增路. 通过切换 $L_{2}^{\prime}$, 我们得到一个最小 $k$-边染色 $\mathcal{C}^{\prime \prime}$ 其中边 $e_{i-1}=v_{i-1} v_{i}$ 仍然染颜色 $i_{1}$ 并且边 $e=v_{i} v_{p}$ 和 $e_{i}=v_{i} v_{i+1}$ 染颜色 $i_{2}$. 同情形 3.1 得出矛盾.

情形 3.3.2 在 $\mathcal{C}^{\prime}$ 中 $E\left(v_{j}\right)$ 的边均不染颜色 $i_{2}$.

此时不妨假设 $E\left(v_{i}\right)$ 中任一边与 $E\left(v_{j}\right)$ 中任一边染互异色. 否则, 出现情形 3.3.1, 可推出矛盾.

因为在 $\mathcal{C}^{\prime}$ 中 $S$ 的两边染同一颜色 $i_{2}$, 设 $e=v_{i} v_{p}$ 和 $f=v_{i} v_{p^{\prime}}$ 染颜色 $i_{2}$, 故在 染色 $\mathcal{C}^{\prime}$ 中 $i_{0}$ 在 $v_{i}$ 不出现, $i_{2}$ 在 $v_{i}$ 至少出现两次. 由引理 3.2 知, $G\left[E_{i_{0}}^{\prime} \cup E_{i_{2}}^{\prime}\right]$ 的含 $v_{i}$ 的连通分支是一个奇圈, 记为 $C_{3}$. 如果 $v^{*} \in V\left(C_{3}\right)$ 或 $\alpha\left(C_{3}, v^{*}, \mathcal{C}^{\prime}\right)<\alpha\left(C_{1}, v^{*}, \mathcal{C}\right)$, 推出矛盾. 否则, $C_{3}$ 必含有另一个顶点 $v_{j^{\prime}}$, 边 $f^{\prime}=v_{j^{\prime}} v_{q^{\prime}}$ 与 $G-V\left(C_{3}\right)$ 的含 $v^{*}$ 的分支 $H^{\prime}$ 关联，其中顶点 $v_{j}$ 在 $H^{\prime}$ 中，则 $d_{i_{2}}\left(v_{i}\right)=2$.

在 $G_{12}$ 中选取以 $e=v_{i} v_{p}$ 作为起始边的最短 $\left(i_{2}, i_{1}\right)$ - 交错链 $L_{3}$, 使其为非增 链并且切换 $L_{3}$ 后, 得到一最小 $k$-边染色 $\mathcal{C}^{\prime \prime}$. 如果 $f=v_{i} v_{p^{\prime}} \notin L_{3}$, 则当切换 $L_{3}$ 后, 在 $\mathcal{C}^{\prime \prime}$ 中边 $e$ 具有颜色 $i_{1}$. 注意到在 $\mathcal{C}^{\prime \prime}$ 中边 $e_{i}$ 和 $e_{i-1}$ 仍然具有颜色 $i_{1}$, 故 $d_{i_{1}}\left(v_{i}\right) \geqslant 3$, 与引理 3.2 矛盾.

现在考虑 $L_{3}$ 含奇圈 $C_{4}=\left(v_{i}, v_{p}, \cdots, v_{p^{\prime}}, v_{i}\right)$ 的情形. 即边 $e$ 和 $f$ 均在 $L_{3}$ 中. 易知 $C_{3} \cup C_{4}$ 为不增链, 但是切换它并不能得到有效的结果. 下面将证明通过寻 找另一个交错链仍然可推出矛盾. 易知在 $\mathcal{C}^{\prime}$ 中边 $g=v_{j} v_{q} \in E\left(v_{j}\right)$ 染颜色 $i_{3}$ 且 $i_{3} \notin\left\{i_{0}, i_{1}, i_{2}\right\}$. 现在选取一条以 $e=v_{i} v_{p}$ 为起始边的 $\left(i_{2}, i_{3}\right)$ - 不增链 $L_{4}$.

(1) 边 $g \notin L_{4}$. 
通过切换 $L_{4}$ 得到一个最小 $k$-边染色 $\mathcal{C}^{\prime \prime}$, 其中边 $g$ 和 $e$ 都具有颜色 $i_{3}$, 则情 形 3.3.1 出现, 推出矛盾.

(2) 边 $g \in L_{4}$ 且边 $f \notin L_{4}$.

通过切换 $L_{4}$, 得到一个最小 $k$-边染色 $\mathcal{C}^{\prime \prime}$, 其中边 $g$ 和 $f$ 均具有颜色 $i_{2}$, 则情 形 3.3.1 出现, 矛盾.

(3) 边 $g$ 和 $f$ 均在 $L_{4}$ 中.

此时由 $L_{4}$ 含边 $g, e$ 和 $f$ 知, $L_{4}$ 包含圈 $C_{5}$. 因为 $G$ 是系列平行图, 故 $C_{1}$ 中 仅有两个顶点 $v_{i}$ 和 $v_{j}$ 与 $H$ 关联. 已知在 $\mathcal{C}^{\prime}$ 中 $E\left(v_{j}\right)$ 的边均不具有颜色 $i_{2}$, 故 3 条边 $g, e$ 和 $f$ 不能同时在 $L_{4}$ 的一个圈中. 如果边 $g$ 和 $e$ 在 $C_{5}$ 中, 则边 $f \notin C_{5}$. 事实上, $C_{5}$ 是一个偶交错圈并且是不增链. 因为 $L_{4}$ 是最短不增链, 有 $L_{4}=C_{5}$ 且 $f \notin C_{5}=L_{4}$, 矛盾, 故必有 $e, f \in C_{5}$ 且 $g \notin C_{5}$. 我们有下面的断言:

断言 2 在 $\mathcal{C}^{\prime}$ 中 $d_{i_{3}}\left(v_{i}\right)=1$.

如果 $d_{i_{3}}\left(v_{i}\right)=0$, 则 $C_{5}$ 为一条非增链与 $L_{4}$ 为最短非增链矛盾. 如果 $d_{i_{3}}\left(v_{i}\right)>$ 1 , 则存在一条边 $h \notin S \cup L_{4}$ 具有颜色 $i_{3}$. 通过切换 $L_{4}$ 得到一个最小 $k$-边染色 $\mathcal{C}^{\prime \prime}$, 其中边 $e, f$ 和 $h$ 具有颜色 $i_{3}$, 故在 $\mathcal{C}^{\prime \prime}$ 中颜色 $i_{0}$ 在 $v_{i}$ 不出现, 颜色 $i_{3}$ 在 $v_{i}$ 至少出 现两次. 由引理 3.2 知, $G\left[E_{i_{0}}^{\prime \prime} \cup E_{i_{3}}^{\prime \prime}\right]$ 的含 $v_{i}$ 的连通分支是一个奇圈, 与在 $\mathcal{C}^{\prime \prime}$ 中 $d_{i_{3}}\left(v_{i}\right) \geqslant 3$ 矛盾. 断言成立.

不妨设 $e^{\prime}=v_{i} v_{r} \in L_{4}$ 具有颜色 $i_{3}$ 并且 $e^{\prime}$ 和 $e_{i}$ 不是平行边. 否则, 可用 $e_{i-1}$ 替换 $e_{i}$. 在 $G_{13}$ 中选取一条以 $e_{i}=v_{i} v_{i+1}$ 为起始边的 $\left(i_{1}, i_{3}\right)$ - 交错链 $L_{5}$, 使得 $L_{5}$ 为不增链并且切换 $L_{5}$ 后可得一个最小 $k$ - 边染色 $\mathcal{C}^{\prime \prime}$. 注意到 $e^{\prime}=v_{i} v_{r} \notin S$. 用 $i_{3}$ 替换 $i_{2}$ 并重复上面的证明. 我们考虑两种情形.

(3.1) $e_{i-1} \notin L_{5}$ 且 $e^{\prime} \notin L_{5}$.

此时 $L_{5}$ 不含包含 $v_{i}$ 的圈. 通过切换 $L_{5}$, 得到一个最小 $k$-边染色 $\mathcal{C}^{\prime \prime}$, 其中边 $e_{i}$ 和 $e^{\prime}$ 均染颜色 $i_{3}$, 故在 $\mathcal{C}^{\prime \prime}$ 中颜色 $i_{0}$ 在 $v_{i}$ 不出现, 颜色 $i_{3}$ 在 $v_{i}$ 至少出现两次. 由引理 3.2 知, $G\left[E_{i_{0}}^{\prime \prime} \cup E_{i_{3}}^{\prime \prime}\right]$ 的含 $v_{i}$ 的连通分支是一个奇圈, 记为 $C_{6}$. 因为在 $\mathcal{C}^{\prime \prime}$ 中 $E\left(v_{i}\right)$ 的边均不具有颜色 $i_{3}$, 从而有 $g \notin C_{6}$ 且 $v_{j} \notin C_{6}$, 故 $C_{6}$ 和 $C_{1}$ 的顶点集不 交. 因为 $H$ 是连通的, $H$ 中存在连接 $v_{q}$ 和 $v_{p}$ 的路 $P^{\prime}$, 故 $P=g \cup P^{\prime} \cup e$ 为 $G$ 中 连接 $v_{j}$ 和 $v_{i}$ 的路. 易知 $C_{1}, C_{6}$ 和 $P$ 构成 $G$ 的 $K_{4}$ 剖分, 与 $G$ 是系列平行图矛 盾.

(3.2) $e_{i-1} \in L_{5}$ 或 $e^{\prime} \in L_{5}$.

此时 $L_{5}$ 含包含顶点 $v_{i}$ 的圈 $C_{7}$. 不妨设 $e_{i-1} \in L_{5}$. 如果 $v_{j} \notin C_{7}$, 由断言 1 的 证明知, $G$ 含 $K_{4}$ 的一个剖分, 矛盾, 故 $v_{j} \in C_{7}$. 如果 $g \in C_{7}$, 则类似于情形 (3.1), $G$ 含 $K_{4}$ 的一个剖分, 矛盾. 如果 $g \notin C_{7}$, 则 $L_{5}$ 的子链 $L_{5}^{\prime}=\left(v_{i}, v_{i+1}, \cdots, v_{j}\right)$ 或 $L_{5}^{\prime}=\left(v_{j}, \cdots, v_{i-1}, v_{i}\right)$ 为不增链, 与 $L_{5}$ 是最小不增链矛盾.

每种情形均推出矛盾. 从而定理得证. 


\section{4 注释与讨论}

易知通过定理的证明过程可获得一个找到该划分的多项式时间算法. 但是 一般情况下, 确定图的不交 $v^{*}$ - 排除边覆盖的最大数目是 $N P$ - 困难的, 因为确 定一个 3 - 连通 3- 正则图 $G$ 是否是 3 - 边可染色的是 $N P$ - 完全的 ${ }^{[12]}$, 这是一般情 形的一个特例. 另一方面, 对平面图还不知确定其不交 $v^{*}$ - 排除边覆盖的最大数 目是否是 $N P$ - 困难的. 但是我们有下面的猜想:

猜想 4.1 设图 $G$ 是一个平面图, 其边连通度为 $\kappa^{\prime}(G)$, 则对任一 $v^{*} \in V(G)$, $E(G)$ 可以划分成 $\min \left\{\kappa^{\prime}(G)+1, \delta(G)\right\}$ 个 $v^{*}$ - 排除边覆盖并且存在找到该划分的 一个多项式时间算法.

可证上述猜想蕴含着四色定理. 众所周知下面的断言蕴含着四色定理 (文献 [16] p.158 定理 9.12).

断言 4.1 任一简单 2-边连通 3-正则平面图是 3- 边可着色的.

下面的断言可从定理 2.1 得到:

断言 4.2 任一简单 2-边连通 3- 正则平面图对任一给定的顶点 $v^{*}$ 均有 3 个 不交 $v^{*}$ - 排除边覆盖.

现在证明断言 4.2 等价于断言 4.1. 显然断言 4.1 蕴含断言 4.2. 假设断言 4.2 成立. 下证断言 4.1 亦成立. 令图 $G$ 为一简单 2 - 边连通 3- 正则平面图, $v^{*}$ 为图 $G$ 的任一给定顶点. 令 $E_{1}, E_{2}$, 和 $E_{3}$ 是 3 个不交 $v^{*}$ - 排除边覆盖, 则每一个 $E_{i}$ 均为边覆盖 $(i=1,2,3)$. 若否, 不失一般性, 设 $d_{1}\left(v^{*}\right)=0$. 但对任一顶点 $v \neq v^{*}$, $d_{i}(v)=1$, 因为图 $G$ 的顶点数为偶数, 有 $\sum_{v \in V(G)} d_{1}(v)$ 为奇数, 矛盾, 故每一 个 $E_{i}$ 都是完美匹配，亦即 $G$ 是 3- 边可着色的.

从上述讨论知，如果一个偶数阶的 $(r-1)$ - 边连通 $r$ - 正则图有 $r$ 个不交 $v^{*}$ 排除边覆盖, 则图 $G$ 亦有 $r$ 个不交边覆盖. 关于一个图的不交边覆盖所构成集 合的最大基数，我们有下面的猜想:

令 $\chi_{c}^{\prime}(G)$ 表示图 $G$ 的不交边覆盖所构成集合的最大基数，也称为图 $G$ 的边 覆盖色数, 即图 $G$ 的 $k$ - 边覆盖染色所用最大颜色数 $k$. 对 $S \subseteq V(G)$, 用 $\lambda(S)$ 表 示至少有一个端点在 $S$ 中的 $G$ 的边所成集合. 在图 $G$ 的任一 $\chi_{c}^{\prime}(G)$ - 边覆盖染色 $\mathcal{C}$ 中, $\lambda(S)$ 中至少有 $\lceil|S| / 2\rceil$ 条边具有相同的颜色, 故 $|\lambda(S)| \geqslant \chi_{c}^{\prime}(G)\lceil|S| / 2\rceil$. 如 果 $|S|$ 为偶数, 则得到界 $\chi_{c}^{\prime}(G) \leqslant \delta(G)$, 但是如果 $|S|$ 为奇数, 可能会提供一些新 的信息. 令

$$
\lambda(G)=\min \left\{\left\lfloor\frac{2|\lambda(S)|}{|S|+1}\right\rfloor: S \subseteq V(G),|S| \geqslant 3 \text { 为奇数 }\right\} .
$$

猜想 4.2 设图 $G$ 为平面图, 则 $\chi_{c}^{\prime}(G)=\min \{\delta(G), \lambda(G)\}$, 并且存在多项式 时间算法找到 $\chi_{c}^{\prime}(G)$.

注意到上述两个猜想对非平面图均不成立. 易证 Petersen 图 ${ }^{[16]}$ 为两个猜想 的反例. 猜想 4.2 同样可推出四色定理, 由此可以看出两个猜想的难度. 但是对 
一些特殊的平面图猜想可能容易解决，并存在找到该种边划分的多项式时间算 法.

\section{参考文献}

1 Coffman E G, Garey Jr M R, Johnson D S, et al. Scheduling file transfers. SIAM J Comput, 1985, 14(3): 326-336

2 Frank A, Gyarfas A. Directed graphs and computer programs. In: Problems Combinatoires et Theorie des Graphes. Collogue Internationaux C N R, 1976, 260: 157-158

3 Lucchesi C L, Younger D H. A minimax theorem for directed graphs. J London Math Soc, 1978, 17(2): 369-374

4 Lovász L. On two minimax theorems in graphs. J Comb Theory Ser B, 1976, 21(1): 96-103

5 Woodall D R, Menger König. Systems in "Theory and applications of graphs" ( Proc Intern Conf, Western Mich Univ). Lecture Notes in Math, 1978, 642: 620-635

6 Feofiloff P, Younger H. Directed cut transversal packing for source-sink connected graphs. Combinatorica, 1987, 7(3): 255-263

7 Schrijver A. Min-max relations for directed graphs. Annals of Discrete Math, 1982, 16(2): 261-280

8 Lee O, Wakabayashi Y. Note on a min-max conjecture of Woodall. J Graph Theorey, 2001, 38(1): 36-41

9 Gupta R P. On decompositions of a multigraph into spanning subgraphs. Bull Amer Math Soc, 1974, 80: 500-502

10 Hilton A J W, de Werra D. A sufficient condition for equitable edge-coloring of simple graphs. Discrete Math, 1994, 128(2): 179-201

11 Miao Lianying, Liu Guizhen. Edge covered coloring and fractional edge covered coloring. J of Systems Science and Complexing, 2002, 15(2): 187-193

12 Seymour P D. Coloring series-parallel graphs. Combinatorica, 1990, 10(4): 379-392

13 Zhou X, Suzuki H, Nishizeki T. A linear algorithm for edge-coloring series-parallel multigraphs. Journal of Algorithms, 1996, 20: 174-201

14 Even G, Naor J, Schieber B, et al. Approximating minimum subset feedback sets in undirected graphs with applications. SIAM J Discrete Math, 2000, 13(2): 255-267

15 Ramachandran V. A minimax arc theorem for reducible flow graphs. SIAM J Discrete Math, 1990, 3: 554-560

16 Bondy J A, Murty U S R. Graph Theory with Application. London: Macmilan Press, 1976. 90-93 